\title{
A Novel Approach to Ising Problems
}

C. Hoede

Department of Applied Mathematics, University of Twente, P.O. Box 217, 7500 AE Enschede, The Netherlands

\section{H.J.W. Zandvliet}

Physical Aspects of Nanoelectronics \& MESA+ Institute for Nanotechnology, P.O. Box 217, 7500 AE Enschede, The Netherlands

\footnotetext{
Abstract

In this note we discuss some of the recent remarks on the solvability of Ising problems. These remarks tend to a conclusion that it is likely that some Ising problems, for example that of the simple cubic lattice, are essentially unsolvable. One type of argument relates such problems to the theory of NP-completeness and intractability. Another type of argument is used to shake the belief that a closed expression for the free energy or a simple equation for the transition temperature is possible. We introduce the idea of gauging on an equation and describe how one might yet find a solution.

PACS numbers: 68.35.Rh Phase transitions and critical phenomena,

75.40.-s Critical point effects, specific heats, short-range order
} 


\section{Introduction}

We consider the Ising model in the ferromagnetic case, i.e. the interaction energies for pairs of neighboring spins $\sigma$ of equal value +1 or -1 are positive, $J>0$. We consider the nearest-neighbor simple square 2D-lattice, the nearest-neighbor 3D-simple cubic lattice and the 2D-simple square lattice with next-nearestneighbor interactions.

For all these lattices the partition function is

$$
Z_{N}=\sum_{\text {states }} e^{-\sum_{n . s .(i, j)} E\left(\sigma_{i}, \sigma_{j}\right) / k_{b} T}
$$

where $N$ is the number of spins, n.s. stands for neighboring spins, $k_{b}$ is Boltzmann's constant and $T$ is the temperature. The summation runs over all $2^{N}$ states of the system and $E\left(\sigma_{i}, \sigma_{j}\right)$ is the interaction energy for neighboring spins $\sigma_{i}$ and $\sigma_{j}$. For the $2 \mathrm{D}$ and $3 \mathrm{D}$ lattices we have interaction energies $J_{X}$ and $J_{y}$, respectively $J_{x}, J_{y}$ and $J_{z}$. For $2 \mathrm{D}+$ next nearest neighbors we have $J_{X}, J_{y}$ and $J_{d}$ for the "diagonal" interactions. We will assume, as is usual, that there are toroidal boundary conditions.

The Ising problem now is to calculate the free energy per spin:

$$
f=\lim _{N \rightarrow \infty} \frac{-k_{b} T}{N} \ln \left(Z_{N}\right)
$$


The calculation for the 2D Ising model, in 1944, by Onsager [1] is really a landmark in the history of Ising problems. He found:

$$
f=\ln 2+\frac{1}{2 \pi^{2}} \int_{0}^{\pi \pi} \int_{0} \ln \left[\operatorname{ch}\left(2 H_{x}\right) \operatorname{ch}\left(2 H_{y}\right)-\operatorname{sh}\left(2 H_{x}\right) \cos (\alpha)-\operatorname{sh}\left(2 H_{y}\right) \cos (\beta)\right] d \alpha d \beta
$$

where $H_{X}=\frac{J_{X}}{k_{b} T}$ and $H_{y}=\frac{J_{y}}{k_{b} T}$. We denote this solution by $O\left(H_{X}, H_{y}\right)$. In case $J_{X}=J_{y}=J$, we write $O(H), H=\frac{J}{k_{b} T}$.

Onsager's algebraic solution was made more transparent by Kaufmann [2] in 1949. $Z_{N}$ is written as $\operatorname{SpurV}{ }^{n} 2$, if $N=n_{1} x n_{2}$, so the $2 \mathrm{D}$ lattice has dimensions $n_{1}$ by $n_{2} . \mathrm{V}$ is a $2^{n_{1}}$ by $2^{n_{1}}$ matrix called the transfer matrix. It is the product of two matrices, one describing the interactions within a column of $n_{1}$ spins and one describing the interactions between two neighboring columns of $n_{1}$ spins. The "bulk calculation" consists of interpreting the matrices as spinor representations of rotation groups, and using that analytical (algebraic) technique to calculate the eigenvalues of V. We stress here the word analytical, as essentially different from numerical, for reasons that will become clearer soon. Having calculated the eigenvalues, the largest one is the important one, one can obtain the closed expression for $f$.

It is worthwhile to note that Onsager gives the reader confidence in his result by pointing out that series development precisely resulted in earlier obtained series developments. 
An important alternative for the bulk calculation was the transformation of $Z_{N}$ into a Pfaffian. In 1961 Kasteleyn [3] first solved the dimer problem, finding a generating function for the numbers of dimers on a 2D lattice, and then showed how this technique could be used to solve the 2D problem too. The Pfaffian constructed, to give $Z_{N}$, did not count all configurations precisely, but a combination of a small finite number of Pfaffians did by compensation of incorrectly counted configurations. For the 3D problem the bulk calculation could be carried out too but the compensation of incorrectly counted configurations required a number of Pfaffians growing with $N$. It was found soon that the technique failed, in that sense, for lattices that were non-planar, like the 3D lattice and the 2D + next nearest neighbor lattice. Analytical techniques for condensing the many Pfaffians into a "closed" formula were not available.

\section{About intractability.}

Recently Istrail [4] has related the difficulties to carry out the bulk calculation for $3 \mathrm{D}$ and $2 \mathrm{D}+$ next nearest neighbors to the theory of NP-complete problems, see Garey and Johnson [5]. In our opinion there is a very basic objection to that. Let us very briefly outline what NP-complete problems are. NP does not stand for Not Polynomial, but for Non-deterministically and Polynomial. There is a class of problems, several thousands by now, that "can be solved in polynomial time by a non-deterministical Turing machine". A potential solution may be offered to the machine and its "truth certificate" can be calculated in polynomial time, i.e. whether the potential solution is correct or not. 
It seems best to give two examples, that make clear that we are considering decision problems really. Their format is as for the Hamiltonian cycle problem.

Given: A graph $\mathrm{G}$ on $\mathrm{N}$ vertices.

Question: Is there a cycle of length N?

A Hamiltonian cycle contains each of the vertices of the graph precisely once, so has the form $v_{1}, v_{2}, \ldots, v_{N}, v_{1}$, where consecutive pairs of vertices form an edge of G. $v_{1}, v_{2}, \ldots, v_{N}$ are labels of the $N$ vertices. The solution is easy in principle. Consider one of the $N$ ! permutations of the labels as potential solution and check whether the edges are present in G. The truth certificate can be calculated in polynomial time. The real difficulty is the "combinatorial explosion", the number $N$ ! of permutations that has to be checked.

One of the first problems considered, see [5], was 3-SAT. A certain expression in $N$ logical variables is given. Is there a truth assignment that makes the expression true? Choosing one of the $2^{N}$ possible truth assignments, the form of the expression guarantees easy checking whether it is a solution. The number $2^{\mathrm{N}}$ presents the essential difficulty again. The problems are intractable. We refer to [5] for the description of NP-completeness, in which 3-SAT plays an important role.

Let us now compare the Ising problem with the intractable problems we just described. On one hand we have an analytical bulk calculation, whereas on the other hand we have an exponential number of cases to check, the combinatorial explosion, for which most probably no shortcut can be found. 
For the intractable problems the nature of a solution is not irrelevant. Rather, it is given from the beginning that it must be a Hamiltonian cycle or a truth assignment. If one would like to see the Ising problem in the setting of the intractable problems, one should consider the situation that somebody comes up with a potential solution and then the problem will be to calculate the truth certificate.

It is here that we recall our remark that Onsager, in a way, gave a truth certificate for his solution by comparing with existing series developments! The comparison of bulk calculation with combinatorial explosion misses the point completely by giving a conclusion that Ising problems like $3 \mathrm{D}$ or $2 \mathrm{D}+$ next nearest neighbors are intractable.

The Ising problem is the calculation of a limit, NP-complete problems are decision problems.

\section{On the transition equation}

For the 2D lattice the critical temperature is described by the transition equation

$\sinh \left(2 H_{X}\right) \sinh \left(2 H_{y}\right)=1$,

conjectured by Kramers and Wannier [6] in 1941. Of course, people have tried to find a transition equation for 3D too, see e.g. Fisher [7], who investigated polynomials $\mathrm{Q}$ in hyperbolic tangents of $H_{X}, H_{y}$ and $H_{Z}$ and potential transition equations of the form $Q=0$. In the last section of [7] doubt is expressed about the possibility of such an equation with the type of polynomials that he considered. 
How bad is this for the hope of finding a way to obtain a solution, or just a transition equation, for the unsolved Ising problems?.

We are strongly reminded of a famous situation in mathematics. Equations of degree 1, 2, 3 and 4 were solvable in terms of root-forms. Most people know the solutions to $a x+b=0$ and $a x^{2}+b x+c=0$, with $x$ as unknown. The result of Galois, and also Abel, that the equations of degree 5 could, in principle, not be solved in terms of root-forms, surds, came as a big shock. But, of course, there are 5 solutions according to the main theorem of algebra.

Now for the transition equation. The $2 \mathrm{D}$ equation can be expressed as a polynomial in hyperbolic tangents and it may be that the $3 \mathrm{D}$ equation, to be found, cannot, in principle. But, there is no objection to a transition equation of essentially deviating form. The problem is only to find such an equation, that should satisfy several conditions that have meanwhile been found in the literature.

Neither the intractability of some problems that can be formulated for nonplanar lattices, see Istrail [4], nor indications that the transition equation would have to have a deviating form from that of the 2D problem, see Fisher [7], are really good reasons to doubt the existence of a closed analytical expression for $f$ in the unsolved cases. However, in the light of what we will present in Section 5, finding the exact transition equation may indeed prove to be a major problem. 


\section{Gauging on an equation}

From our discussion of intractability we retain two things. First, there is the idea of truth certificate. If somebody comes up with a potential solution, we should be able to decide on the quality of that solution. The obvious check is to see whether the solution gives the results of series developments. For the 3D Ising model these are known for more than half a century. We recall our remark made before about Onsager. Second, it seems that structural aspects, like non-planarity of the lattice, do indeed form obstacles for any technique of analytical treatment of the bulk calculation. Instead of trying to explain why such a calculation is not possible in principle, we suggest to skip the bulk calculation altogether and try to make an argumented guess about potential solutions.

This suggestion, at first sight, seems preposterous. How to guess Onsager's solution for $2 \mathrm{D}$ ? However, the $2 \mathrm{D}$ solution $O\left(H_{X}, H_{y}\right)$ being known, the situation is different. We can look upon both the 3D model and the $2 \mathrm{D}+$ next nearest neighbor model as 2D models with adjusted interaction energies.

In its clearest form this is the case for the $2 \mathrm{D}+$ next nearest neighbor model. If the diagonal interactions between next-nearest neighbors vanish, we regain the 2Dmodel, for which the solution is known. Also the 3D-model reduces to the 2Dmodel in case one of the interaction energies is going to zero and the lattice decouples into an array of 2D lattices. For the 2D + next nearest neighbor model this also happens if the nearest-neighbor interactions vanish. The lattice decouples into two interpenetrating 2D lattices. We will not go into the interesting phenomena occurring in that case here. 
It is clear that any solution $O\left(H_{X}, H_{y}, H_{d}\right)$ for the $2 \mathrm{D}+$ next nearest neighbor model should yield Onsager's solution $O\left(H_{x}, H_{y}\right)$ for $2 \mathrm{D}$, if $H_{d} \rightarrow 0$. Likewise a potential solution $O\left(H_{X}, H_{y}, H_{Z}\right)$ should yield that solution for $H_{Z} \rightarrow 0$. However, the bulk calculation is missing. It is here that the transition equation starts to play an important role. It is on that equation that we would want to gauge the potential solution.

The transition equation for the 2D model is a thread to hold on to. Let us denote it by $T E\left(H_{x}, H_{y}\right)$. Assuming that both 2D + next nearest neighbor and 3D model can be simulated by a 2D model with adjusted interaction energies, so that the potential solution would be $O\left(H_{x}^{*}, H_{y}^{*}\right)$, we face the problem to express $H_{X}^{*}$ and $H_{y}^{*}$ in terms of $H_{X}, H_{y}$ and $H_{d}$ respectively $H_{X}, H_{y}$ and $H_{z}$. The crux of our proposal now is to use the transition equation to do this. That is why we speak of gauging on an equation. We want to simulate the unsolved problems by the solved 2D problem. Having found $H_{X}^{*}$ and $H_{y}^{*}$ in terms of $H_{X}, H_{y}$ and $H_{d}$ respectively $H_{X}, H_{y}$ and $H_{Z}$ we have found argumented potential solutions.

The simulating 2D model has transition equation $\operatorname{TE}\left(H_{X}^{*}, H_{y}^{*}\right)$. The simulated 2D + next nearest neighbor model or 3D-model can be expected to have transition equation $T E\left(H_{X}, H_{y}, H_{d}\right)$ respectively $T E\left(H_{X}, H_{y}, H_{z}\right)$. For $H_{d} \rightarrow 0$ respectively $H_{Z} \rightarrow 0$ these equations should yield $T E\left(H_{X}, H_{y}\right)$ for the $2 \mathrm{D}$ model. The approach should now be clear: 
(a) Find a transition equation $T E\left(H_{X}, H_{y}, H_{d}\right)$, respectively $\operatorname{TE}\left(H_{X}, H_{y}, H_{z}\right)$.

(b) Use this equation to express $H_{X}^{*}$ and $H_{y}^{*}$ in terms of $H_{x}, H_{y}$ and $H_{d}$ respectively $H_{X}, H_{y}$ and $H_{z}$ to obtain potential solutions $O\left(H_{x}^{*}, H_{y}^{*}\right)$.

(c) Check the result with series developments to obtain a truth certificate.

In this approach the bulk calculation is circumvented at the cost of

(i) Not being completely sure about the truth certificate. The known series developments are very extensive, but they might be recovered from $O\left(H_{X}^{*}, H_{y}^{*}\right)$ and yet the potential solution might be slightly wrong.

(ii) The necessity to find a correct transition equation for the unsolved cases.

The approach outlined here arose as reaction to a recent result of Zandvliet [8] for the $2 \mathrm{D}+$ next nearest neighbor model. Using a domain wall free energy calculation he derived the following $T E\left(H_{X}, H_{y}, H_{d}\right)$;

$e^{-2 H_{X}}+e^{-2 H_{y}}+e^{-2\left(H_{X}+H_{y}\right)}\left(2-e^{-4 H_{d}}\right)=e^{4 H_{d}}$.

For $H_{d} \rightarrow 0$ this equation reduces to

$e^{-2 H_{X}}+e^{-2 H_{y}}+e^{-2\left(H_{x}+H_{y}\right)}=1$,

which can be transformed into the more familiar form $\sinh \left(2 H_{X}\right) \sinh \left(2 H_{y}\right)=1$. 
As gauging equation, in the isotropic case, we now have

$2 e^{-2 H^{*}}+e^{-4 H^{*}}=2 e^{-2 H-4 H_{d}}+e^{-4 H}\left(2 e^{-4 H_{d}}-e^{-8 H_{d}}\right)$.

This equation expresses $H^{*}$ in terms of $H$ and $H_{d}$. The potential solution is then $O\left(H^{*}\right)$. Hoede and Zandvliet [9] investigated the transition equation for the isotropic $2 \mathrm{D}+$ next nearest neighbor model and found that $O\left(H^{*}\right)$ indeed had the right properties near $H_{d}=0$ (for a weak next neighbor coupling one finds $H^{*}=H+\sqrt{H_{d}}$, which should be property of the exact solution [10]). They also found a transition equation that fitted the known Monte Carlo results very well. Yet not all demands to be posed on the transition equation, in particular the behavior near $H=0$, the decoupling situation, were satisfied.

For the 3D model the transition equation has been subject to extensive studies. We already mentioned Fisher's paper. We recently found the following transition equation for the anisotropic 3D Ising model $\left(H_{Z}>>H_{X, y}\right)$;

$\sinh \left(2 H_{z}\right) \sinh \left(2 H_{x}+2 H_{y}\right)=1$.

This equation is only valid in a restricted area of the phase diagram. Here we have another example of gauging. With $H_{x}^{*}=H_{z}$ and $H_{y}^{*}=H_{x}+H_{y}$ we obtain

$\sinh \left(2 H_{x}^{*}\right) \sinh \left(2 H_{y}^{*}\right)=1$

With $H_{x}^{*} \gg H_{y}^{*}$, this yields $O\left(H_{x}^{*}, H_{y}^{*}\right)$, the correct solution for the quasi-onedimensional model. 
Equation (2) reads

$\sinh \left(2 H_{z}\right)\left(\sinh \left(2 H_{x}\right) \cosh \left(2 H_{y}\right)+\sinh \left(2 H_{y}\right) \cosh \left(2 H_{x}\right)\right)=1$

This suggests that the transition equation for the 3D-model might have the form

$\sinh \left(2 H_{X}\right) \sinh \left(2 H_{y}\right) \cosh \left(2 H_{Z}\right)+\sinh \left(2 H_{X}\right) \sinh \left(2 H_{Z}\right) \cosh \left(2 H_{y}\right)+$ $\sinh \left(2 H_{y}\right) \sinh \left(2 H_{Z}\right) \cosh \left(2 H_{X}\right)+C\left(H_{X}, H_{y}, H_{Z}\right)=1$

The correction term $C\left(H_{X}, H_{y}, H_{Z}\right)$ should vanish whenever $H_{X}, H_{y}$ or $H_{Z}$ is put equal to 0 . It might for example contain a factor $\sinh \left(2 H_{X}\right) \sinh \left(2 H_{y}\right) \sinh \left(2 H_{Z}\right)$. Such a condition stems from the demand that in all three cases the transition equation $T E\left(H_{X}, H_{y}\right), T E\left(H_{X}, H_{Z}\right)$ respectively $T E\left(H_{y}, H_{z}\right)$ should be recovered. There are several other demands, like the correct transition point in the isotropic case, which is extremely well known from Monte Carlo calculations [11], or the behavior for the 3D to 2D transition.

All such demands pose problems for the transition equation, but in view of the approach proposed in this paper it seems worth while to tackle them. To illustrate the idea we just mention the equation

$$
\begin{aligned}
& \sinh \left(2 H_{X}\right) \sinh \left(2 H_{y}\right)+\sinh \left(2 H_{y}\right) \sinh \left(2 H_{Z}\right)+\sinh \left(2 H_{X}\right) \sinh \left(2 H_{Z}\right)+ \\
& 4\left(1-\frac{1}{2} e^{-4\left(H_{X}+H_{y}+H_{Z}\right)}\right) \sinh \left(2 H_{X}\right) \sinh \left(2 H_{y}\right) \sinh \left(2 H_{Z}\right)=1
\end{aligned} .
$$


The equation gives the 2D transition equation if any of the $H_{X}, H_{y}$ and $H_{Z}$ is zero. For $H_{Z} \rightarrow 0$ we recover equation (1). It is symmetrical in $H_{X}, H_{y}$ and $H_{Z}$ and in the isotropic case this equation yields $H_{C}=0.2216549$, which should be compared with $H_{C}=0.2216544$ given in ref. [11] for the critical point. This equation lacks an argumentation, e.g. a domain wall free energy calculation, and also other demands have not been checked. We only use it to illustrate the idea of gauging on an equation. The gauging equation would be

$\sinh ^{2}\left(2 H^{*}\right)=3 \sinh ^{2}(2 H)+4\left(1-\frac{1}{2} e^{-12 H}\right) \sinh ^{3}(2 H)$.

This determines $H^{*}(H)$ and the potential solution $O\left(H^{*}\right)$ for the isotropic 3D problem. We did not investigate the series development in order to get a truth certificate, mainly because we are in doubt about equation (3) as far as the other demands are concerned.

\section{Discussion}

We have given two examples of gauging equations. Another simple example can be given for the antiferromagnetic $2 \mathrm{D}$ model with external magnetic field. Müller-Hartmann and Zittartz [12] conjectured the equation

$\sinh \left(2 H_{X}\right) \sinh \left(2 H_{y}\right)=\cosh \left(H_{\mu}\right)$, 
as transition equation for that model. Here $H_{\mu}=\frac{\mu M}{k_{b} T}$, with magnetic moment $\mu$ and external magnetic field M. For $M \rightarrow 0, \cosh \left(H_{\mu}\right) \rightarrow 1$ and we regain Equation (1). The gauging equation would now be, for the isotropic case,

$$
\frac{\sinh ^{2}(2 H)}{\cosh \left(H_{\mu}\right)}=\sinh ^{2}\left(2 H^{*}\right)
$$

yielding $H^{*}\left(H, H_{\mu}\right)$ and potential solution $O\left(H^{*}\right)$.

If the idea of gauging is valid, we can indeed focus on the transition equation and circumvent a bulk calculation. However, we are in need of some support for the idea of gauging. We will focus on the isotropic case. From Sloane's dictionary of integer sequences [13] we found the high temperature series developments in $y=\tanh \left(\mathrm{H}^{*}\right)$ and $\mathrm{x}=\tanh (\mathrm{H})$, for the magnetic susceptibility of the $2 \mathrm{D}$ and $3 \mathrm{D}$ model respectively, as given in Table I.

In case the 3D model can be simulated by the 2D model these series should have the same value. We do not consider the first term! The reversion of the remaining series in $y$, see e.g. Abramowitz and Stegun 3.6.25 [14], yields $y$ as a series in $f(x)$, where $f(x)$ is the series in $x$, without the first term. Substitution then yields a series $S(x)$ for $y$ expressed in $x$. This is a gauging equation, $y=S(x)$, that follows from the decades old work on series developments, see Sloane [13] for the references. The result was the series given in Table II, where also successive estimates of the 3D-transition value are given. 


\begin{tabular}{|l|l|}
\hline $2 D$ & $3 D$ \\
\hline 1 & 1 \\
\hline$+4 y$ & $+6 x$ \\
\hline$+12 y^{2}$ & $+30 x^{2}$ \\
\hline$+36 y^{3}$ & $+150 x^{3}$ \\
\hline$+100 y^{4}$ & $+726 x^{4}$ \\
\hline$+276 y^{5}$ & $+3510 x^{5}$ \\
\hline$+740 y^{6}$ & $+16710 x^{6}$ \\
\hline$+1972 y^{7}$ & $+79494 x^{7}$ \\
\hline$+5172 y^{8}$ & $+375174 x^{8}$ \\
\hline$+13492 y^{9}$ & $+1769686 x^{9}$ \\
\hline$+34876 y^{10}$ & $+8306862 x^{10}$ \\
\hline$+89764 y^{11}$ & $+38975286 x^{11}$ \\
\hline$+229628 y^{12}$ & $+182265822 x^{12}$ \\
\hline$+585508 y^{13}$ & $+852063558 x^{13}$ \\
\hline$+1486308 y^{14}$ & $+3973784886 x^{14}$ \\
\hline$+3763460 y^{15}$ & $+18527532310 x^{15}$ \\
\hline$+9497380 y^{16}$ & $+86228667894 x^{16}$ \\
\hline$+23918708 y^{17}$ & $+401225368086 x^{17}$ \\
\hline$+60080156 y^{18}$ & $+1864308847838 x^{18}$ \\
\hline$+150660388 y^{19}$ & $+8660961643254 x^{19}$ \\
\hline$+377009364 y^{20}$ & $+40190947325670 x^{20}$ \\
\hline$+942106116 y^{21}$ & $+186475398518726 x^{21}$ \\
\hline
\end{tabular}

Table I

High temperature series for the magnetic susceptibility 


\begin{tabular}{|c|c|}
\hline$y=3\left(\frac{x}{2}\right)$ & $H_{1}=0.2835$ \\
\hline$+3\left(\frac{x}{2}\right)^{2}$ & $H 2=0.2510$ \\
\hline$+3\left(\frac{x}{2}\right)^{3}$ & $H_{3}=0.2479$ \\
\hline$+69\left(\frac{x}{2}\right)^{4}$ & $H_{4}=0.2406$ \\
\hline$+459\left(\frac{x}{2}\right)^{5}$ & $H_{5}=0.2358$ \\
\hline$+411\left(\frac{x}{2}\right)^{6}$ & $H_{6}=0.2354$ \\
\hline$+22791\left(\frac{x}{2}\right)^{7}$ & $H_{7}=0.2327$ \\
\hline$+66213\left(\frac{x}{2}\right)^{8}$ & $H_{8}=0.2319$ \\
\hline$+1154723\left(\frac{x}{2}\right)^{9}$ & $H_{9}=0.2305$ \\
\hline$+3786801\left(\frac{x}{2}\right)^{10}$ & $H_{10}=0.2300$ \\
\hline$+69728919\left(\frac{x}{2}\right)^{11}$ & $H_{11}=0.2290$ \\
\hline
\end{tabular}




\begin{tabular}{|c|c|}
\hline$+236351403\left(\frac{x}{2}\right)^{12}$ & $H_{12}=0.2286$ \\
\hline$+4391519223\left(\frac{x}{2}\right)^{13}$ & $H_{13}=0.2279$ \\
\hline$+14977375443\left(\frac{x}{2}\right)^{14}$ & $H_{14}=0.2277$ \\
\hline$+290669917619\left(\frac{x}{2}\right)^{15}$ & $H_{15}=0.2271$ \\
\hline$+1017776595693\left(\frac{x}{2}\right)^{16}$ & $H_{16}=0.2269$ \\
\hline$+19566870142959\left(\frac{x}{2}\right)^{17}$ & $H_{17}=0.2265$ \\
\hline$+71084173367165\left(\frac{x}{2}\right)^{18}$ & $H_{18}=0.2263$ \\
\hline$+1357967026040259\left(\frac{x}{2}\right)^{19}$ & $H_{19}=0.2260$ \\
\hline$+5017309562668557\left(\frac{x}{2}\right)^{20}$ & $H_{20}=0.2259$ \\
\hline$+96031088694860083\left(\frac{x}{2}\right)^{21}$ & $H_{21}=0.2256$ \\
\hline
\end{tabular}

Table II

3D solution function $S(x)$ and successive approximations of the critical point 
Now in particular the 3D critical temperature should correspond with the 2D critical temperature, i.e. from $H_{C}^{*}$ should follow $H_{C}$. As $y=\tanh \left(H^{*}\right)$, we know that $y_{C}=\sqrt{2}-1$. Giving $y$ that value, we can now solve for $x_{C}$ and $\operatorname{arctanh}\left(x_{C}\right)$ gives $H_{C}$ for the $3 \mathrm{D}$ model. As a first example we just take the first term $\frac{3}{2} x$. Then $\sqrt{2}-1=\frac{3}{2} x$, so $x=\frac{2}{3}(\sqrt{2}-1)=0.27614 \ldots$ and $H_{1}$, the first approximation to $H_{C}$, is $0.2835 \ldots$ Taking the first two terms gives $H_{2}=0.2510 \ldots$, etc.

We see that the approximations indeed tend to the value 0.221654 .. mentioned earlier as best known result from Monte Carlo calculations. For our purpose it is this tendency that gives the support for our idea that the 3D model can indeed be simulated by the $2 \mathrm{D}$ model. Any transition equation leading to a gauging equation should be consistent with the series found for $y$ in terms of $x$. This rules out Equation (3), in spite of the fact that it gave a very accurate value for the transition point and had some other desired properties.

The form of the solution function, $S(x)$, makes it unlikely that indeed a "nice" transition equation can be found, in line with the findings of Fisher [7].

Nevertheless, solving $y=S(x)$ we find $H^{*}=\operatorname{arctanh}(S(x))$ and substitution of $H^{*}$ for $H$ in $O(H)(\equiv O(H, H))$ gives an approximate solution $O\left(H^{*}\right)$ to the $3 \mathrm{D}$ problem as result of our approach.

\section{Acknowledgment}

We thank P.H.M. Kersten for programming the reversion. 


\section{References}

[1]. L. Onsager, Phys Rev. 65, 117 (1944).

[2]. B. Kaufmann, Phys. Rev. 76, 1232 (1949) and B. Kaufmann and L. Onsager, Phys. Rev. 76, 1244 (1949).

[3]. P. Kasteleyn, Physica 27, 1209 (1961).

[4]. S. Istrail, Statistical mechanics, three-dimensionality and NP completeness. In: Proceedings of the annual ACM symposium on the theory of computing (STA), 87-96 (2000).

[5]. M.R. Garey and D.S. Johnson, Computers and Intractability, Freeman, San Francisco (1979).

[6]. H.A. Kramers and G.H. Wannier, Phys. Rev. 60, 252-263 (1941).

[7]. M.E. Fisher, J. Phys.A: Math. Gen. 28, 6323-6333 (1995).

[8]. H.J.W. Zandvliet, Europhys. Letters 73, 747 (2006), see also H.J.W. Zandvliet, Rev. Mod Phys. 72, 593 (2000).

[9]. C. Hoede and H.J.W. Zandvliet, On Ising models, Memorandum No. 1804, Department of Applied Mathematics, University of Twente, ISBN. 0169-2690 (2006).

[10]. T.W. Burkhardt, Z. Physik B 31, 183-186 (1978).

[11]. H.W.J. Blöte, E. Luijten and J.R. Heringa, J. Phys.A: Math. Gen. 28, 6289-6313 (1995).

[12]. E. Müller-Hartmann and J. Zittartz, Z. Physik B 27, 261 (1977).

[13]. M.J.A. Sloane, The on-line Encyclopedia of Integer Sequences, http://www.research.att.com/ njas/sequences/.

[14]. M. Abramowitz and I.A. Stegun, Handbook of Mathematical Functions, Dover Publications, New York (1965). 